\title{
Panorama de la seguridad del paciente en los laboratorios clínicos de México
}

\author{
Overview of patient safety in clinical laboratories of Mexico \\ Eduardo Mucito-Varela*
}

* Investigador

independiente, M en C

Bioquímicas, Químico

Bacteriólogo Parasitólogo.

\section{Correspondencia: EMV}

eduardomucito@gmail. com

Conflicto de intereses:

El autor declara

que no tiene.

Citar como: Mucito-

Varela E. Panorama

de la seguridad

del paciente en los

laboratorios clínicos

de México. Rev

CONAMED 2020; 25(1):

34-46.

doi: $10.35366 / 92893$

Financiamiento:

Investigación financiada

por el mismo autor.

Recibido: 22/11/2019.

Aceptado: 31/03/2020.

\section{RESUMEN}

Los laboratorios clínicos son un componente vital de los servicios de salud, pues abordan a los pacientes durante todo el proceso de atención. No obstante, el impacto real de los errores del laboratorio en la seguridad del paciente es desconocido, debido a que existe escasa investigación y registros sobre los errores que suceden en el proceso total de análisis de los laboratorios en México. El cumplimiento de la NOM-007-SSA3-2011 permite garantizar el cumplimiento de las Acciones esenciales para la seguridad del paciente, aunque es necesario implementar actividades adicionales para prevenir errores y coadyuvar en el proceso de medicación segura. En esta revisión se muestra un panorama sobre la situación de la seguridad del paciente en los laboratorios clínicos de México y se proponen estrategias para abordar los errores de laboratorio y fomentar la cultura de seguridad del paciente.

Palabras clave: Seguridad del paciente, servicios de laboratorio clínico, calidad de la atención de salud, errores diagnósticos, errores médicos, errores de medicación.

\begin{abstract}
Clinical laboratories are a vital component of health services because they approach to patients throughout the entire care process. However, the real impact of laboratory errors on patient safety is unknown, because there is little research and records about errors that occur in the total analysis process of laboratories in Mexico. Compliance with the essential actions for patient safety stated by Mexican government, leads us to analyze the degree to which we can cover the patient's safety requirements with the Official Mexican Regulations, to detect opportunities for improvement and ensure the safety of patients using laboratory diagnostic services. In this review, a compilation of the most frequent types of laboratory errors in the literature, proposals to address them and some strategies to promote the culture of patient safety in clinical laboratories in Mexico are presented.
\end{abstract}

Keywords: Patient safety, clinical laboratory services, quality of health care, diagnostic error, medical errors, medication errors. 


\section{INTRODUCCIÓN}

La atención sanitaria está conformada por todos los servicios que reciben las personas o las comunidades para promover, mantener, vigilar o restablecer la salud. El proceso de atención resulta de una compleja interacción de profesionales de distintas áreas, procesos y tecnologías, que aunque siempre tienen la mejor intención hacia con el paciente, no son infalibles y por lo tanto no están exentos de fallar.' Adicionalmente, la creciente complejidad de la economía de los sistemas de salud, de los métodos diagnósticos que emplean tecnologías de alta especialización y los cambios en la estructura demográfica de las poblaciones representan riesgos emergentes que afectan la seguridad de los pacientes. ${ }^{2}$ Entonces, podemos decir que existe siempre un riesgo latente para los pacientes que tiene que ser controlado para prevenir o minimizar los daños.

Para controlar la calidad de la atención sanitaria se han desarrollado diferentes estrategias, pero fue hasta 2004 cuando apareció el movimiento de Seguridad del Paciente, impulsado por la Organización Mundial de la Salud.' Este movimiento surge ante la creciente evidencia de la pobre calidad de la atención en los sistemas de salud, que comenzó con la publicación de las estadísticas de los Institutos de Medicina (IOM) de Estados Unidos a finales de los noventas en el multicitado estudio «To err is human». ${ }^{3}$ Desde entonces, el movimiento de Seguridad del Paciente se ha fortalecido durante las dos últimas décadas con el apoyo de las redes sanitarias de diferentes países.

La investigación en calidad de la atención nos ha permitido detectar que los incidentes relacionados con la seguridad del paciente, que incluyen a los eventos adversos, tienen su origen en los errores médicos. Estos errores surgen de la interacción de diferentes elementos organizacionales, de infraestructura, de diseño y control de procesos, así como culturales y del contexto en el que se brinda la atención. ${ }^{4}$ Quizá las equivocaciones médicas más comunes son los errores en el diagnóstico, que incluyen falla y retraso en el mismo. ${ }^{4}$ Estos desaciertos pueden deberse al fallo para ordenar los estudios adecuados, uso de estudio o tratamiento fuera de uso y falla para actuar de acuerdo a los resultados de estudios o de monitoreo. ${ }^{5}$
En México es obligatoria la implementación del documento Acciones esenciales para la seguridad del paciente, que permite el monitoreo y la prevención de eventos adversos de los integrantes del Sistema Nacional de Salud. ${ }^{6}$ Como parte del Sistema Nacional de Salud, Ios laboratorios clínicos son establecimientos del sector público o privado que a través del análisis de muestras biológicas aportan evidencia para confirmar o realizar un diagnóstico, prevenir o dar seguimiento a una enfermedad y monitorear un tratamiento. ${ }^{7}$ Considerando que cerca de $70 \%$ de las decisiones médicas están basadas en los resultados proporcionados por los laboratorios clínicos, ${ }^{8}$ es importante reconocer el impacto de los errores del laboratorio en la seguridad del paciente. Sin embargo, en México existe poca investigación sobre la frecuencia de eventos adversos y errores del laboratorio clínico. ${ }^{9}$ Por este motivo se realizó esta revisión bibliográfica, para recopilar la información sobre los tipos de eventos adversos que suceden en otros países como consecuencia de los errores del laboratorio clínico y así poder diseñar un marco de referencia que permita mejorar a los laboratorios clínicos de México a través de la investigación y la aplicación de las acciones esenciales para la seguridad del paciente.

\section{EVENTOS ADVERSOS ASOCIADOS AL USO DEL LABORATORIO CLÍNICO}

De acuerdo a los reportes en la literatura, aproximadamente 3\% de los eventos adversos que ocurren en los servicios de atención sanitaria están asociados al uso de pruebas diagnósticas, siendo la frecuencia de hasta $4.23 \%$ en hospitales pequeños..$^{10}$ Los eventos adversos asociados al uso del laboratorio clínico pueden tener su origen en errores ocurridos en cualquiera de las fases del proceso total de análisis (Tabla 7). Algunos estudios estiman que de 24 a 30\% de los errores ocurridos durante el proceso total de análisis repercuten en la atención del paciente, aunque sólo de 3 a 12\% se traducen en eventos adversos, siendo la mayoría cuasiincidentes que de haber ocurrido tendrían probabilidades de daño elevadas; sin embargo, más de 80\% de esos errores pueden ser evitables.10,11

El impacto real de los errores de laboratorio sobre la seguridad del paciente se desconoce, debido a 


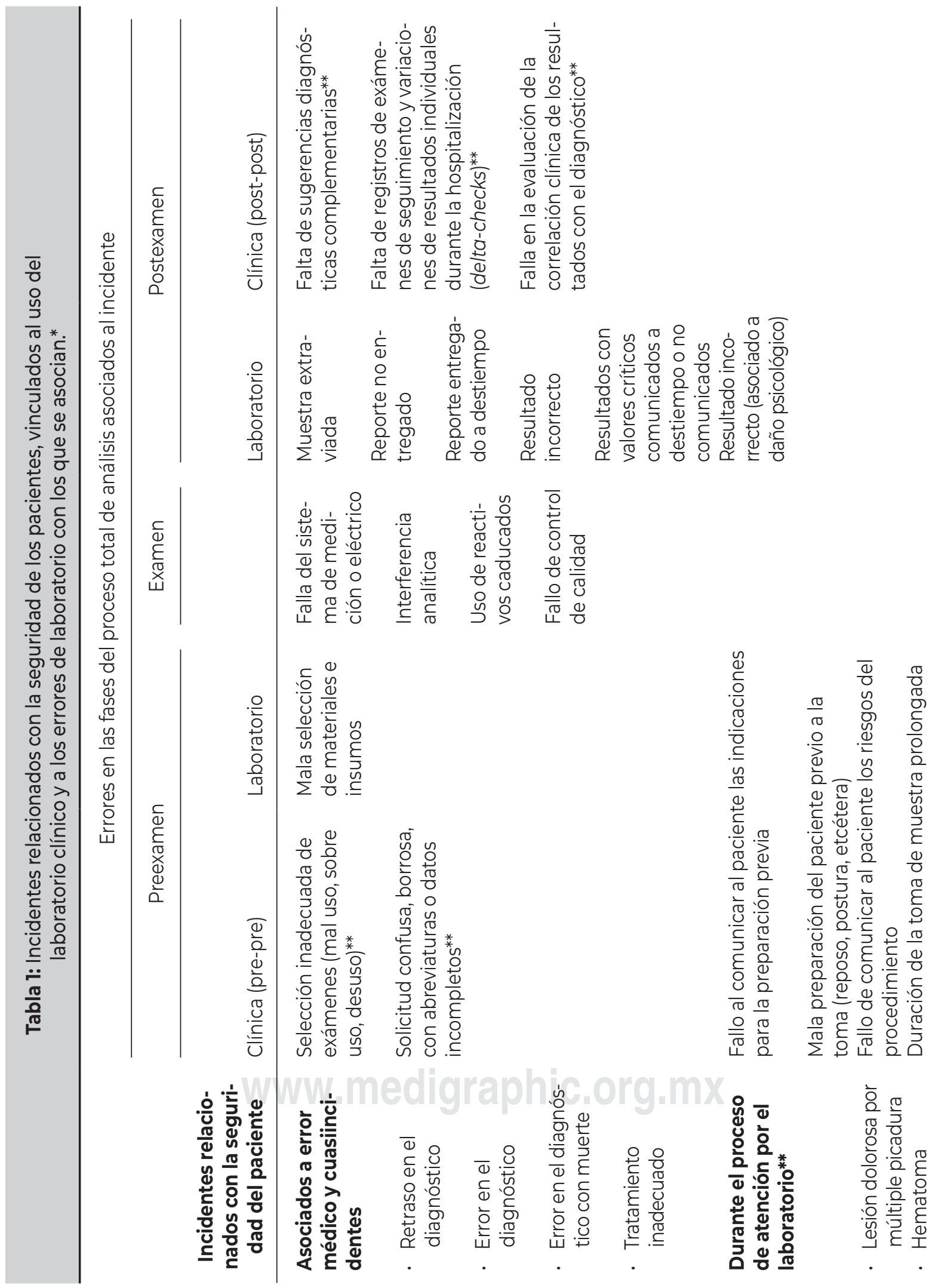




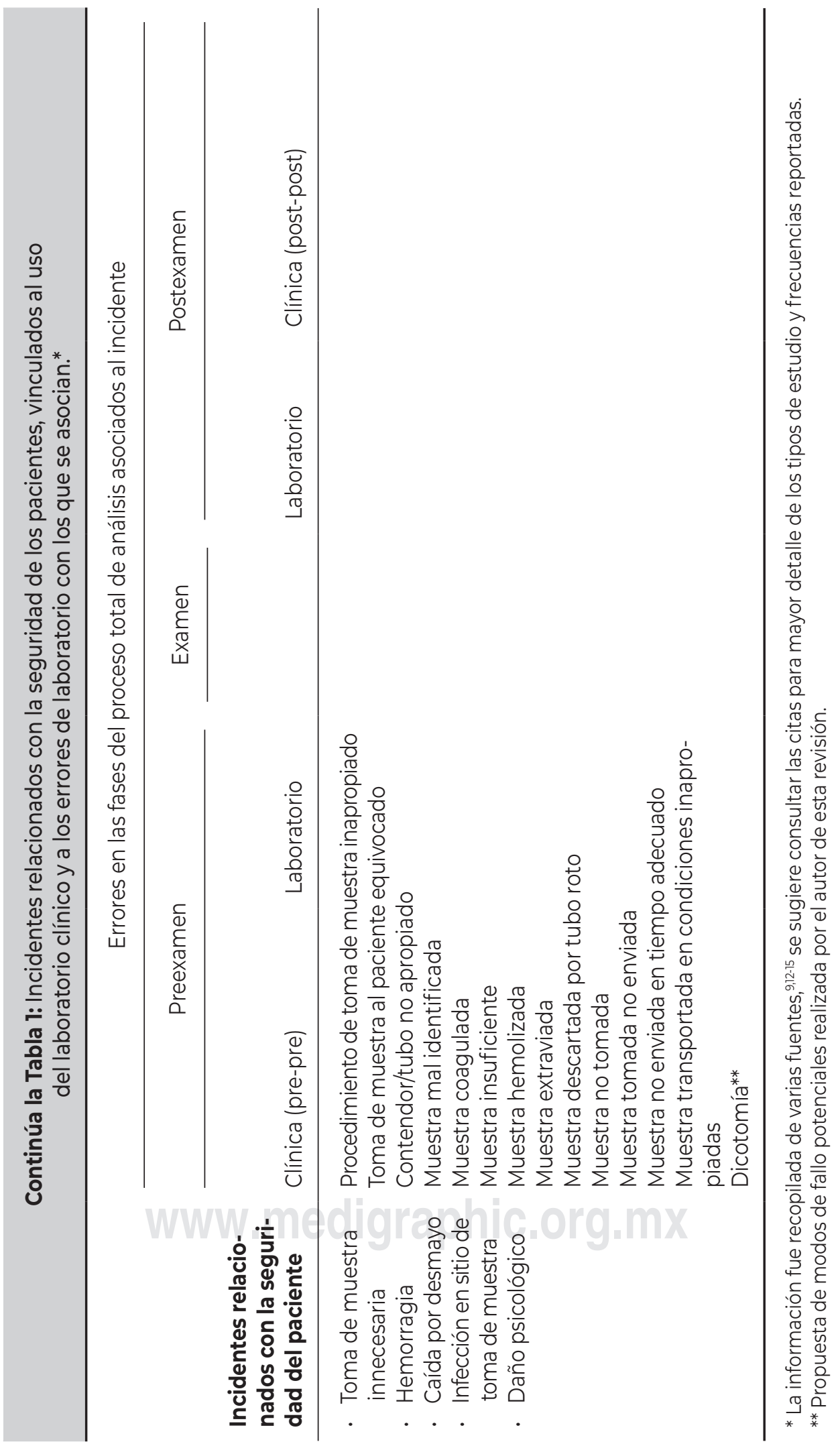


que existen pocos registros de incidentes ocurridos en el laboratorio clínico o como consecuencia de los errores del laboratorio, ${ }^{9,12}$ sobre todo de las etapas pre-y postexamen en la interfase médico-laboratorio y del lado clínico, que son las que presentan una mayor frecuencia de errores..$^{13}$ Una de las razones por las que se podrían subestimar los incidentes ocurridos como consecuencia de los errores de laboratorio, es la falta de investigación y notificación obligatoria de los errores e incidentes ocurridos en los laboratorios. También podemos agregar que, en países en los que los laboratorios clínicos pueden funcionar como organizaciones del sector privado, los pacientes pueden acudir libremente a solicitar un examen de laboratorio sin orden médica, lo cual aumenta el riesgo de daños innecesarios o que generan algunas lesiones que no se podrían considerar graves como hematomas, lesión dolorosa en el sitio de punción, caídas por desmayo, etcétera, y que pasan inadvertidos si el paciente no se queja o porque tiene elección para cambiar a otro laboratorio donde considere que será mejor atendido. Sin embargo, debemos considerar que un daño asociado a la atención sanitaria se considera como cualquier tipo de alteración estructural, funcional y sus consecuencias, lo cual incluye enfermedad, lesión, sufrimiento, discapacidad y muerte. ${ }^{16}$

Algunos errores de laboratorio se han considerado de mayor prioridad por su frecuencia de recurrencia elevada y riesgo de daño asociado. ${ }^{14,17}$ Para lograr conocer más acerca de los errores de laboratorio y su impacto en la seguridad del paciente, la International Federation of Clinical Chemistry and Laboratory Medicine (IFCC) ha propuesto el uso de indicadores de calidad armonizados que permitan monitorear los errores en los procesos intra- y extraanalíticos del laboratorio, de manera que sea fácil su comparación y evaluación en diferentes partes del mundo. ${ }^{18}$ No obstante, cada laboratorio debe adoptar los indicadores de calidad que le permitan satisfacer las necesidades de sus pacientes, de los médicos y de las autoridades sanitarias y regulatorias, con resultados confiables y oportunos. ${ }^{19}$

\section{LA SEGURIDAD DEL PACIENTE EN LOS LABORATORIOS CLÍNICOS DE MÉXICO}

En México existen 13,453 laboratorios de análisis clínicos, reportados en la base de datos del Directorio
Estadístico Nacional de Unidades Económicas (DENUE) del INEGI. De este total, 100 laboratorios corresponden al sector público y 13,353 al sector privado (https://www.inegi.org.mx/app/mapa/ denue/, consultado: 28-octubre-2019). Los requisitos oficiales regulatorios para la operación de un laboratorio clínico están establecidos en la Norma Oficial Mexicana NOM-007-SSA3-2017, Para la organización y funcionamiento de los laboratorios clínicos.7 Algunos autores han propuesto que el cumplimiento de la normatividad oficial es una estrategia para lograr objetivos de calidad asistencial y seguridad del paciente. ${ }^{20}$ Aunque la NOM007-SSA3-2011 no tiene un apartado explícito para la seguridad del paciente, contiene elementos de utilidad para garantizar siete de las ocho acciones esenciales para la seguridad del paciente. Sin embargo, es necesario reforzar algunas actividades e integrar nuevos requisitos en la normatividad oficial para alcanzar los objetivos de seguridad del paciente de manera efectiva (Tabla 2).

«La seguridad en el proceso de medicación» es una acción esencial no cubierta en la NOM007-SSA3-2011, a pesar de que la propia definición de la actividad del laboratorio clínico en dicha norma contempla el apoyo en el tratamiento de las enfermedades y que, de acuerdo a la NOM220-SSA1-2016 para la instalación y operación de la farmacovigilancia, las reacciones adversas de un medicamento pueden identificarse mediante estudios de laboratorio. ${ }^{21}$ Las recomendaciones a la NOM-007-SSA3-2011 sugeridas en este trabajo tienen fundamento en la evidencia generada de la investigación en seguridad del paciente, así como en los requisitos establecidos en la NMX-EC15189-IMNC-2015/ISO 15189:2012,22 que es la norma mediante la cual se acreditan los laboratorios clínicos para comparar su calidad y competencia de manera internacional. Además, se sugiere complementar con requisitos para la tecnovigilancia y farmacovigilancia, pues la identificación de eventos adversos asociados al uso de dispositivos médicos y errores de medicación son áreas que competen a los laboratorios clínicos. ${ }^{21,23}$

Un aspecto muy importante a implementar es la cultura de seguridad del paciente (CSP), que se puede considerar como el grado en el que un grupo de trabajo se involucra para lograr los objetivos de seguridad del paciente y que se puede 


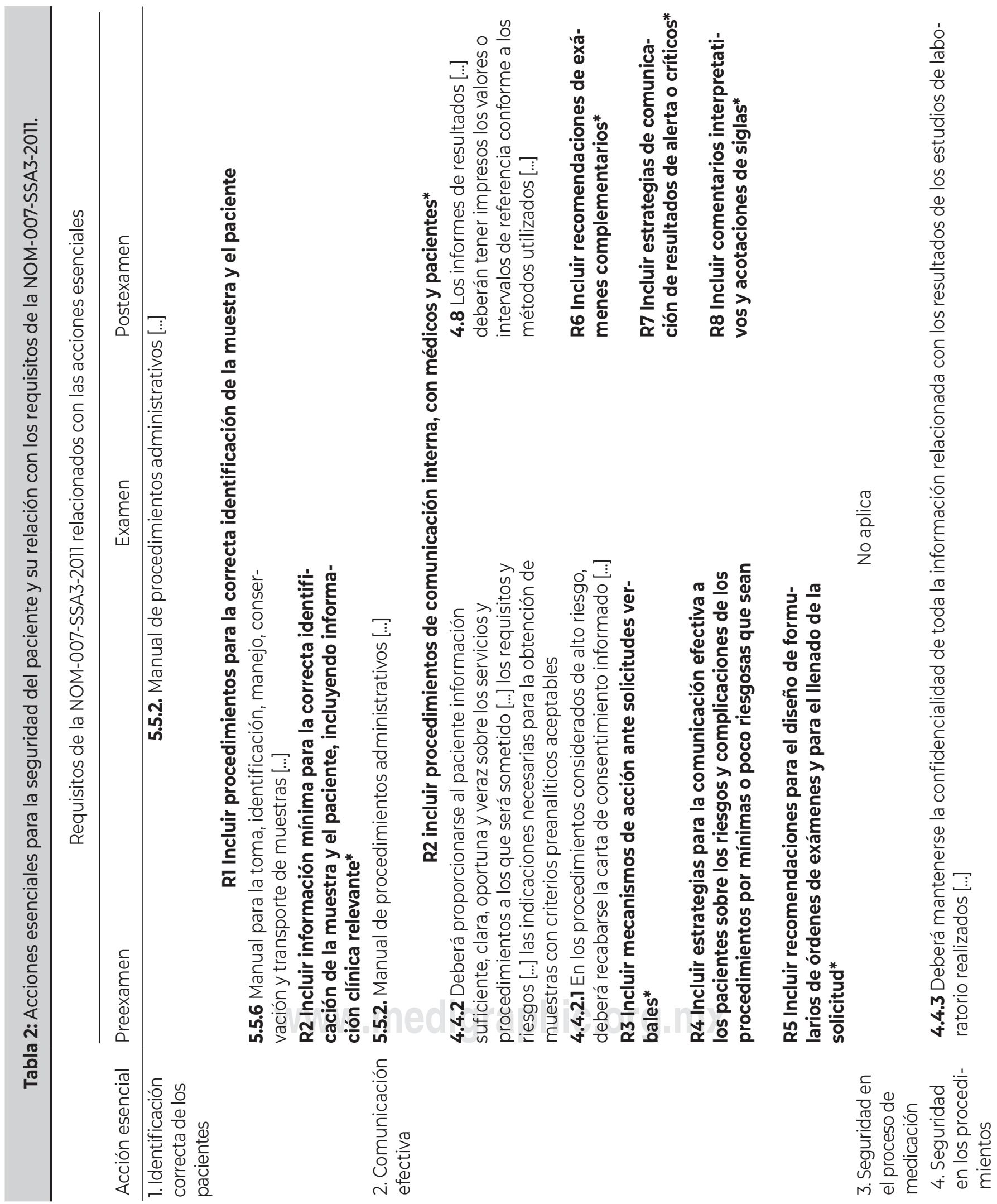




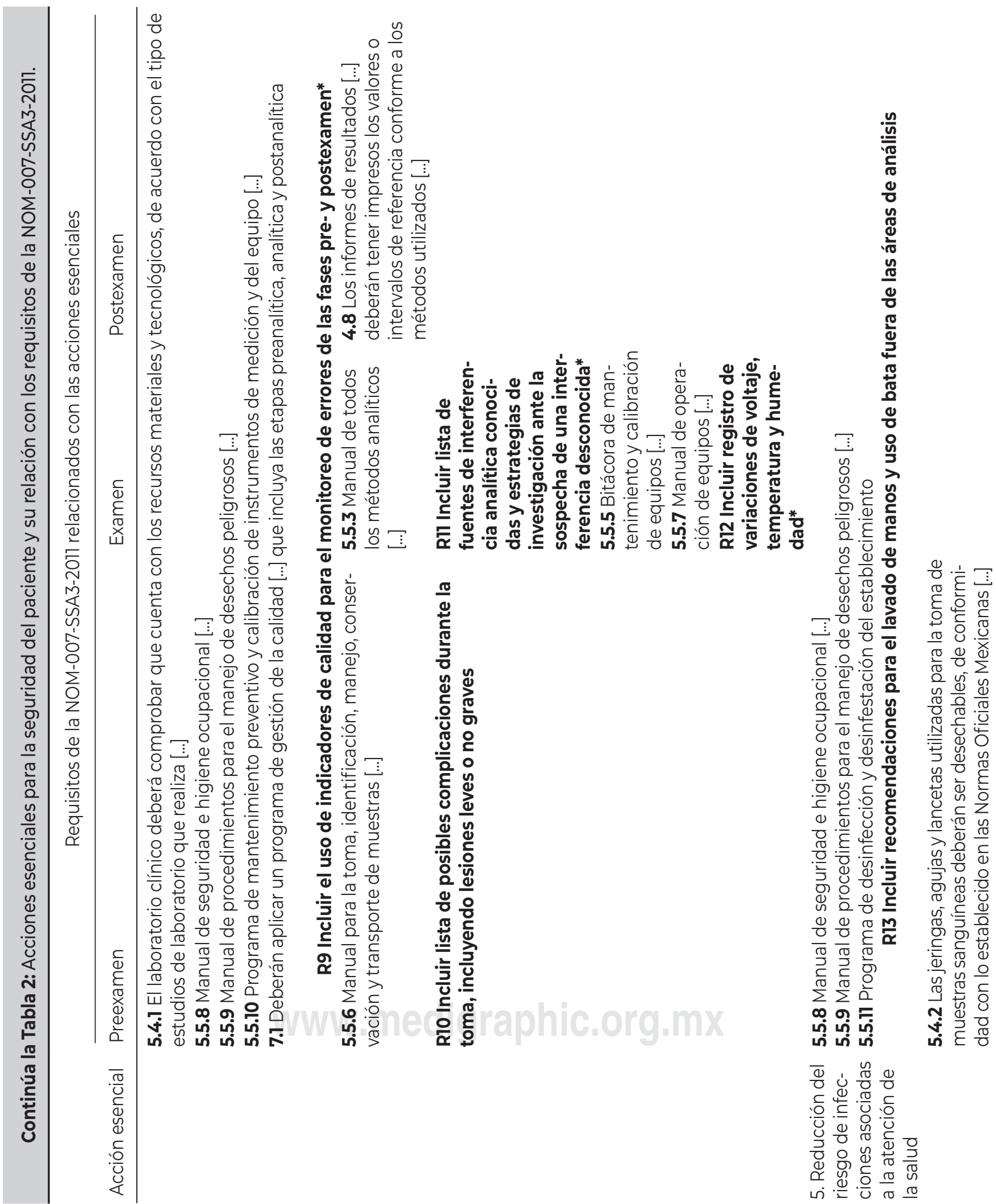




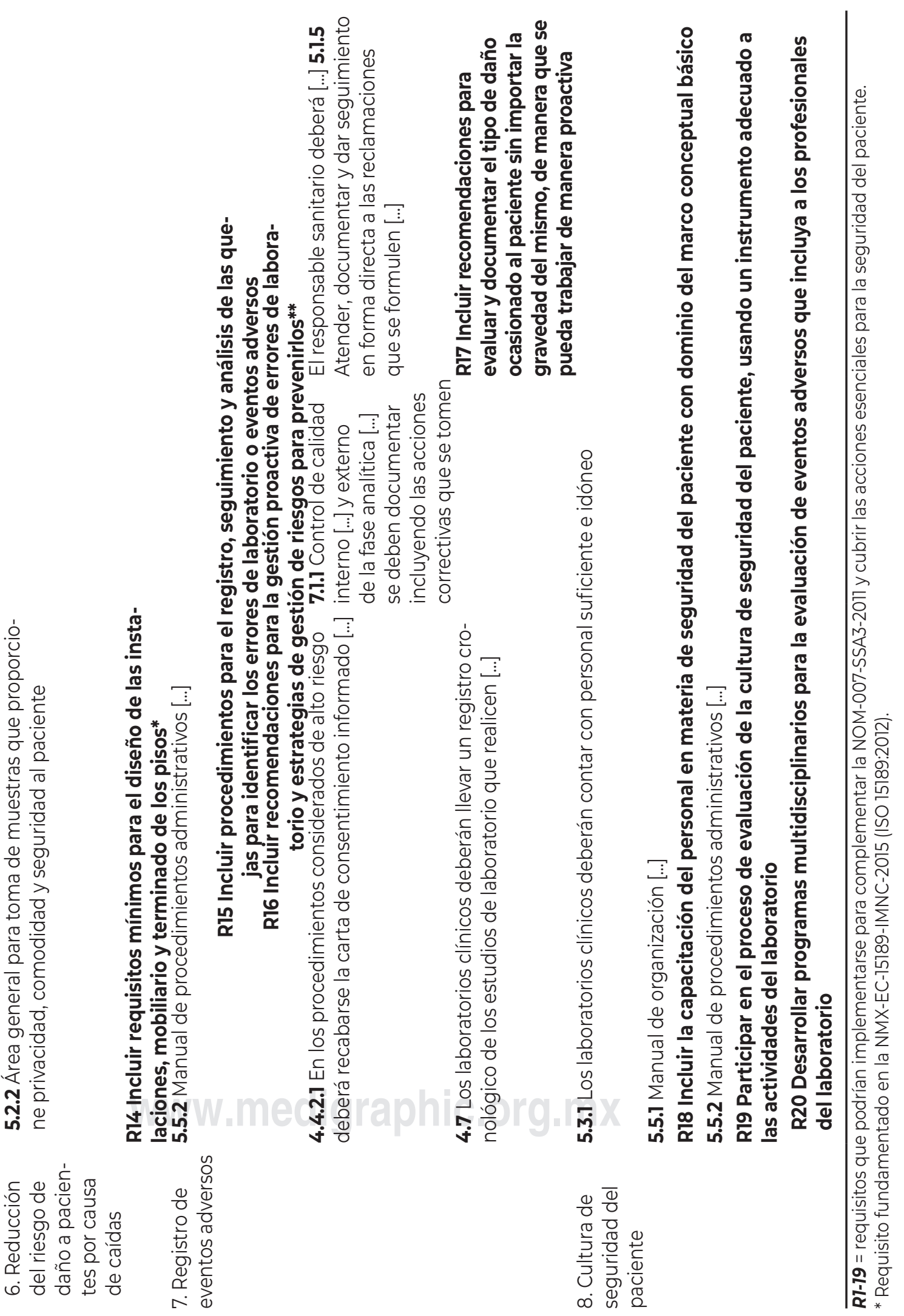


expresar como un conjunto de percepciones, conocimientos, habilidades y actitudes del personal en aspectos que abarcan la seguridad del sujeto (notificación de errores, trabajo en equipo, liderazgo). ${ }^{24} \mathrm{El}$ documento de Acciones esenciales para la seguridad del paciente propone como estrategia para evaluar la CSP el empleo de un cuestionario estandarizado, mismo que ha sido utilizado en diferentes hospitales de México. ${ }^{25}$ En dichos estudios, el personal del laboratorio clínico no ha sido considerado, además de que no contempla requisitos particulares del proceso total de análisis de los laboratorios clínicos.

Una propuesta para evaluar la CSP en los laboratorios clínicos es emplear un cuestionario adaptado a las actividades propias del laboratorio clínico. Giménez-Marín y colegas diseñaron y validaron un instrumento con el que entrevistaron satisfactoriamente en 2015 a 740 trabajadores de laboratorios clínicos de 27 hospitales públicos en España, lo que les permitió detectar áreas de oportunidad para mejorar el trabajo diario enfocado en garantizar la seguridad de los pacientes. ${ }^{24}$ Este instrumento fue adaptado y se invitó a profesionales del laboratorio clínico a contestarlo a través de Facebook, durante septiembre de 2017 a enero de 2018 (https://forms.gle/RrQr3ivuhNEqgCtY7). Obtuvimos una participación estimada de 36\% al considerar que 42 profesionales de laboratorio clínico contestaron la encuesta y la liga obtuvo 115 clics, aunque fue visto por cerca de 4,000 personas. Las características demográficas y laborales de los participantes de la encuesta se muestran en la (Tabla 3); a pesar de la poca respuesta que obtuvimos logramos validar el cuestionario en nuestra población, pues obtuvimos un valor alfa de Cronbach de 0.94 (Tabla 4). Resulta de gran interés que en concordancia al estudio de Giménez-Marín y colaboradores, ${ }^{24}$ los trabajadores del laboratorio clínico están sensibilizados hacia la seguridad de los pacientes, pues se sienten orgullosos de su trabajo y reconocen que sus acciones pueden generar errores con riesgo de afectar a pacientes. Aunque de manera global, todos los aspectos evaluados representan áreas de oportunidad para mejorar, ya que obtuvieron puntajes positivos con menos de $75 \%$ de respuesta positiva promedio (Tabla 5). Los ítems con puntaje más bajo sugieren que se debe trabajar en mejorar la calidad de los procesos

\begin{tabular}{lc}
\multicolumn{2}{c}{ Tabla 3: Características demográficas y } \\
ocupacionales de los profesionales de laboratorio \\
clínico encuestados con el instrumento. \\
\multicolumn{2}{c}{ Evaluación de la cultura de seguridad } \\
del paciente en el laboratorio clínico.
\end{tabular}

preexamen y examen realizados manualmente, así como reforzar el compromiso de los jefes de laboratorio para atender los asuntos relacionados a la seguridad de los pacientes, al emplear una postura no punitiva que fomente el aprendizaje colaborativo a partir de los errores.

Para garantizar que los profesionales de la salud se gradúen de las universidades con competencias 
para la seguridad del paciente, la Organización Mundial de la Salud publicó la Guía curricular sobre seguridad del paciente. ${ }^{26}$ La implementación en las escuelas de medicina, odontología, enfermería y farmacia han permitido desarrollar estrategias de enseñanza exitosas, así como generar cambios en las actitudes y prácticas de los estudiantes universitarios y recién graduados que salen a la práctica clínica. ${ }^{24}$ Aunque en México estas estrategias se empiezan a implementar en la formación de médicos, odontólogos y enfermeras, ${ }^{27}$ la formación de químicos especializados en áreas de la medicina de laboratorio, quienes son generalmente los responsables de los laboratorios clínicos, presenta currículos con tópicos sobre seguridad del paciente que se cubren de manera aislada en diferentes cursos. Adicionalmente, asociaciones civiles como la «Red Mexicana de pacientes por la seguridad del paciente» ofertan cursos de capacitación a profesionales de la salud. Sin embargo, aún hace falta evidencia para evaluar las competencias y la cultura de seguridad del paciente durante la formación de profesionales de laboratorio, por lo que se plantea la adecuación de los planes de estudio de las carreras relacionadas con el diagnóstico clínico y farmacia para integrar una asignatura obligatoria que introduzca los aspectos esenciales de seguridad del paciente, en la que se fomenten la comunicación y el trabajo en equipo multidisciplinario y que permita desarrollar gradualmente las competencias requeridas para la seguridad del paciente conforme a la guía curricular de la OMS.26
Una fortaleza actual de los laboratorios clínicos es su experiencia en la implementación en sistemas de gestión de calidad e innovación en sus procesos. La cultura de calidad se fomenta en los profesionales de los laboratorios desde hace ya varias décadas y debería ser aprovechada para impulsar la seguridad del paciente. Por supuesto, es necesario prestar mayor esfuerzo a las fases prey postexamen, pues en la actualidad contamos con estrategias de control total de la calidad bien aceptadas y estandarizadas para la fase de análisis. En este sentido, debemos priorizar acciones para el reporte y registro de los errores de laboratorio, que permitan la investigación y la evaluación del impacto en el proceso de atención, sin minimizar los daños ocasionados a los pacientes. Para esto, sería de gran utilidad incorporar el uso de indicadores de calidad armonizados internacionalmente para monitorear los errores de laboratorio durante todas las fases del proceso total de análisis.

La certificación y acreditación de los sistemas de calidad en los laboratorios clínicos podría ser una estrategia eficaz que permita incorporar el uso de indicadores que impactan en la seguridad del paciente, pues la aplicación de los principios fundamentales de calidad generan un marco organizacional que permite la sistematización de procesos indispensables para minimizar los errores como son: el liderazgo, la comunicación, la documentación y la gestión de riesgos. ${ }^{22}$

Aunque hay un gran avance en la certificación y acreditación de los laboratorios clínicos, aún existe un gran porcentaje de laboratorios de los que no

Tabla 4: Análisis de la confiabilidad del cuestionario. Evaluación de la cultura de seguridad del paciente en el laboratorio clínico*.

\begin{tabular}{lc}
\hline Parámetro & Resultado \\
\hline Coeficiente alfa con las puntuaciones observadas & 0.94 \\
Coeficiente alfa con las puntuaciones estandarizadas & 0.95 \\
Lambda 6 de Guttman & 1.00 \\
Correlación promedio entre los ítems & 0.24 \\
Razón señal/ruido & 19.00 \\
Intervalo de confianza 95\% & $0.92-0.97$
\end{tabular}

* El cuestionario consta de 62 ítems (preguntas), los ítems 23, 25, 28, 51 y 58 son ítems con escala invertida. Para el análisis estadístico se empleó la función «alpha» dentro de la paquetería «Psych» de R.27,28 


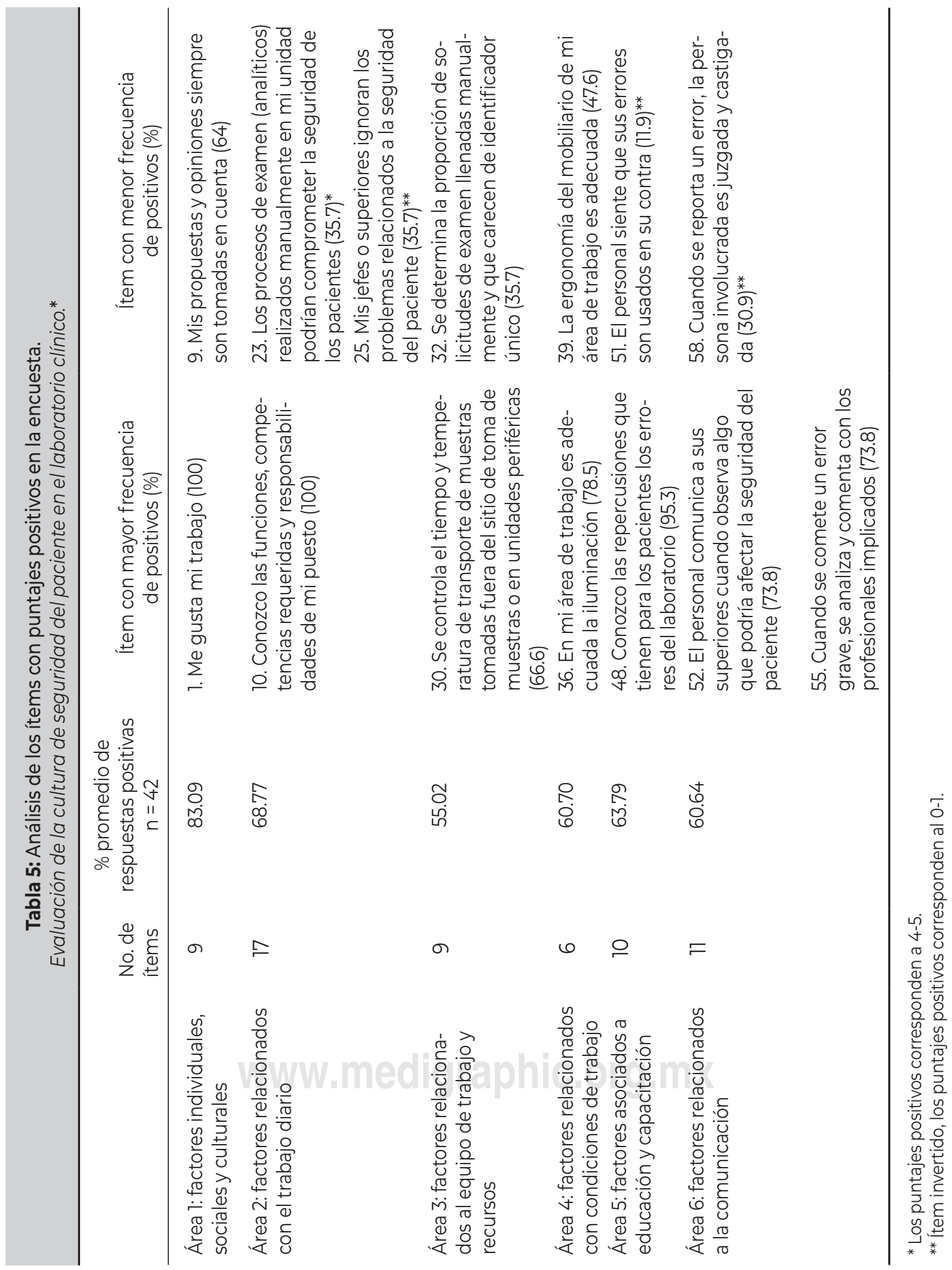


tenemos referente en cuanto a su calidad y competencia. Por ejemplo, en el padrón de laboratorios acreditados bajo ISO 15189 de la Entidad Mexicana de Acreditación ((http://consultaema.mx:75/Directorio_CL/Principal.aspx). Acceso: 29-octubre-2019)), se cuenta con un total de 141 laboratorios acreditados, lo que representa apenas el 1\% de los laboratorios del país. Cabe resaltar que laboratorios del sector público se comienzan a acreditar, lo que representa un gran avance en la calidad de la atención. Puesto que la acreditación es un proceso opcional para los laboratorios, el cumplimiento de la NOM-007-SSA3-2011 podría fungir como elemento clave para las auditorias de calidad y seguridad del paciente, aunque no hay registro público que permita identificar la proporción de laboratorios clínicos que han aprobado por proceso de evaluación de la conformidad bajo esta norma oficial.

\section{CONCLUSIONES}

El laboratorio clínico puede asumir un papel más activo en el cuidado del paciente, más allá de las cuatro paredes de sus instalaciones, al capacitar e interconsultar a médicos, enfermeras y otros profesionales de la salud. Por lo que debemos de integrar a los profesionales del laboratorio en grupos multidisciplinarios para trabajar por la seguridad y bienestar de los pacientes. Para estos fines es necesario fomentar la capacitación y educación en materia de seguridad del paciente en todos los profesionales de la salud, incluyendo a los profesionales de los laboratorios clínicos desde su formación profesional. Adicionalmente, la medición de la CSP en los profesionales del laboratorio, la notificación obligatoria de los errores de laboratorio y la investigación de eventos adversos nos permitirá identificar los procesos que se deben mejorar con prioridad para alcanzar los objetivos de atención segura de los pacientes.

Una meta a corto plazo para la seguridad del paciente es fomentar el uso apropiado del laboratorio clínico, incluyendo la selección, el uso adecuado de las pruebas diagnósticas, el informe oportuno de los resultados y la interpretación de los mismos. Esto involucra que los laboratorios clínicos trabajen en conjunto con las autoridades regulatorias y administrativas de clínicas y hospitales para el diseño de procesos de atención más eficientes, en los que se haga notoria la experiencia de los profesionales especializados en el laboratorio clínico.

Finalmente, para alcanzar los objetivos de seguridad del paciente y avanzar a la par de otros servicios de salud, es necesario aplicar de manera efectiva y adecuar la normatividad oficial aplicable a los laboratorios clínicos. Asimismo, los retos actuales para los laboratorios clínicos son la implementación de sistemas de notificación de errores e incidentes, así como de indicadores de calidad enfocados a la seguridad del paciente en todos sus procesos. Esto debe incluir sistemas de tecnovigilancia de los dispositivos médicos y pruebas diagnósticas empleadas, además de la implementación de procesos de soporte para la farmacovigilancia que facilitarán lograr la meta de medicación segura. Por último, la inclusión de los pacientes como elementos clave de su propia seguridad implica educar y concientizar a la población en materia de seguridad del paciente y sobre el uso de las pruebas diagnósticas.

\section{Biblografía}

1. Lippi G, Simundic AM, Mattiuzzi C. Overview on patient safety in healthcare and laboratory diagnostics. Biochem Medica. 2010; 20 (2): 131-143.

2. Yu A, Flott K, Chainani N, Fontana G, Darzi A. Patient Safety 2030. 2016. Available in: http://www.imperial.ac.uk/media/ imperial-college/institute-of-global-health-innovation/centrefor-health-policy/Patient-Safety-2030-Report-VFinal.pdf.

3. Kohn LT, Corrigan JM, Donaldson MS. To err is human: builta safer health system. Vol. 126, National Academy Press. 1999.

4. Carver N, Gupta V, Hipskind EJ. Medical error. In: StatPearls [Internet]. Treasure Island, Florida: StatPearls Publishing, 2019 [Consulted 2019 May 15]. Available in: https://www.ncbi. nlm.nih.gov/books/NBK430763/.

5. Vázquez-Frías JA, Villalba-Oriz P, Villalba-Caloca J, MontielFalcón H, Hurtado-Reyes C. El error en la práctica médica. ¿Qué sabemos al respecto? An Med (Mex) [Serie en Internet] 2011 [Consultado 2019 octubre 30]; 56 (1): 49-57. Disponible en: http://www.medigraphic.com/pdfs/abc/ bc-2017/bc111j.pdf.

6. Consejo de Salubridad General. ACUERDO por el que se declara la obligatoriedad de la implementación, para todos los integrantes del Sistema Nacional de Salud, del documento denominado Acciones Esenciales para la Seguridad del Paciente. [Internet]. México: Diario Oficial de la Federación; 2017 [Consultado 2019 octubre 30]. Disponible en: http://www.dof.gob.mx/nota_detalle.php? codigo $=5496728 \&$ fecha $=08 \% 2 F 09 \% 2 F 2017$. 
7. Secretaría de Salud. NORMA Oficial Mexicana NOM-007SSA3-2011, Para la organización y funcionamiento de los laboratorios clínicos. México: Diario Oficial de la Federación;2012.

8. Forsman RW. Why is the laboratory an afterthought for managed care organizations? Clin Chem. 1996; 42: 813-816.

9. Angüiano-Sánchez NV, Perales-Quintana MM, DíazOlachea CC, Cázares-Tamez R, Pérez-Chávez F, Llaca-Díaz JM. Errores en el laboratorio clínico; evaluación de tipos y frecuencias. Med Univ. 2011; 13 (52): 133-138.

10. Aranaz-Andrés JM, Aibar-Remón C, Vitaller-Burillo J, RuizLópez P. Estudio Nacional sobre los Efectos Adversos ligados a la Hospitalización. ENEAS 2005 [Internet]. Madrid, España: Ministerio de Sanidad y Consumo, 2006 [Consultado 2019 octubre 30]. Disponible en: http://www. msssi.gob.es/organizacion/sns/planCalidadSNS/pdf/ excelencia/opsc_sp2.pdf.

11. Hawkins R. Managing the pre- and post-analytical phases of the total testing process. Ann Lab Med. 2012; 32 (1): 5-16.

12. Agius $\mathrm{C}$, Buhagiar $\mathrm{C}$. Deciphering an unexpected serum creatinine result in a critically ill patient. J Appl Lab Med An AACC Publ. 2017; 2 (3): 430-435.

13. Plebani $\mathrm{M}$. The detection and prevention of errors in laboratory medicine. Ann Clin Biochem. 2010; 47: 101-110.

14. Astion ML, Shojania KG, Hamill TR, Kim S, Ng VL. Classifying laboratory incident reports to identify problems that jeopardize patient safety. Am J Clin Pathol. 2003; 120 (1): 18-26.

15. Straseski JA, Strathmann FG. Patient data algorithms. Clin Lab Med [Internet]. 2013; 33 (1): 147-160. Available in: http:// dx.doi.org/10.1016/j.cll.2012.11.009.

16. Organización Mundial de la Salud. Más que palabras. Marco Conceptual de la Clasificación Internacional para la Seguridad del Paciente. Informe Técnico Definitivo Enero de 2009. OMS [Internet], 2009; 1-160. Disponible en: http://www.who.int/patientsafety/implementation/icps/ icps_full_report_es.pdf.

17. Lao EG, García ÁS, Figuerola MB, Moreno E, Paraire AH. Errors of clinical laboratory and its impact on patient safety. Open J Soc Sci. 2017; 5 (3): 243-253.

18. Sciacovelli L, Lippi G, Sumarac Z, West J, Garcia Del Pino Castro I, Furtado Vieira K et al. Quality Indicators in
Laboratory Medicine: the status of the progress of IFCC Working Group "laboratory Errors and Patient Safety" project. Clin Chem Lab Med. 2017; 55 (3): 348-357.

19. Barth JH. Clinical quality indicators in laboratory medicine. Ann Clin Biochem. 2012; 49 (1): 9-16.

20. Gutiérrez A. Las Normas Oficiales como un elemento de control de la Calidad y Seguridad del paciente. Boletín CONAMED-OPS. 2015; 20-22.

21. Secretaría de Salud. NORMA Oficial Mexicana NOM-220SSAT-2016, Instalación y operación de la farmacovigilancia. México: Diario Oficial de la Federación; 2017.

22. NMX-EC-15189-IMNC-2015 (ISO 15189:2012). Laboratorios Clínicos-Requisitos particulares de la calidad y competencia. Instituto Mexicano de Normalización y Certificación, A.C., 2015.

23. Secretaría de Salud. NORMA Oficial Mexicana NOM-240SSA1-2012, Instalación y operación de la tecnovigilancia. México: Diario Oficial de la Federación; 2012.

24. Giménez-Marín A, Rivas-Ruiz F, García-Raja AM, VentaObaya R, Fusté-Ventosa M, Caballé-Martín I et al. Assessment of patient safety culture in clinical laboratories in the Spanish national health system. Biochem Med (Zagreb). 2015; 25 (3): 363-376.

25. Castro y Vázquez PJ, Tanguma RD, España RI. Una visión de la cultura de seguridad del paciente en hospitales en México. Rev CONAMED [Internet]. 2016; 21: (3): 109120. Disponible en: https://www.medigraphic.com/pdfs/ conamed/con-2016/con763b.pdf.

26. World Health Organization. Multi-professional patient safety curriculum guide. Edition. Paitent Saf. 2011, pp. 1-272.

27. Farley $D$, Zheng $H$, Rousi E, Leotsakos A. Evaluation of the WHO Multi-Professional Patient Safety Curriculum Guide. 2013. Available in: http://www.who.int/patientsafety/ education/curriculum/EN_PSP_Curriculum_Evaluation/ en/.

28. Revelle W. Package "psych"-procedures for psychological, psychometric and personality research. R Packag [Internet]. 2015, pp. 1-358. Available in: http://personalityproject.org/r/psych-manual.pdf.

\section{NOTA ACLARATORIA}

En el artículo Depresión en madres médicas en México: un estudio transversal, Volumen 24 Suplemento 1, 2019, la filiación de Karen

Rosales-Mariscal es : Neonatóloga. Hospital Español de México. Facultad Mexicana de Medicina, Universidad La Salle México. 